\title{
Importance of Pollution Controlling by Grey Correlation Analysis
}

\author{
Li-na WANG ${ }^{1^{*}}$, Yi-tong WANG ${ }^{2}$ \\ ${ }^{a, 1}$ Management School, Liaoning University of Technology, Jinzhou 121001, Liaoning, China \\ ${ }^{\mathrm{a}, 1}$ Economy \& Management School, Hainan Normal University, Haikou 571158, Hainan, China \\ ${ }^{2}$ Radio and Film School, Liaoning University, Shenyang 110000, Liaoning, China
}

*Corresponding author: Lina Wang, PhD., e-mail address: lina1976113@126.com

\begin{abstract}
Key words: Pollution controlling; Importance of indicators; Grey association analysis; Incentives and supervision; Importance
\end{abstract}

Abstract:Pollution controlling's incentive and monitoring are important issues in environmental management. However, in the mechanism of pollution controlling, the importance of pollution controlling indexes determine the selection of controlling order and efficiency of pollution controlling, management authorities, to sewage enterprises' pollution-control behavior, often demanding evidence and identify which ones are more important incentive and monitoring indicators. 3 pollution controlling's Incentive and monitor indicators are selected: pollution controlling quality, pollution controlling costs, on-time completion rate, setting 4 sets of variable factors, modeling and analysis based on grey correlation theory and doing research, concluded that the relative importance of indicators, expected to offer reference for effectively motivating enterprises to control pollution and supervising authorities.

\section{Introduction}

Pollution control is an important environmental management work. The latest progress in pollution control are as follows: study on specifications: JoschkaGerigk ${ }^{1}$ established a standard model, validity of the revised environmental standards. Kimitoshi Sato ${ }^{2}$ builds on the overall energy balance of the global climate warming and urban models, and examines the climate change factors of human genes. $\mathrm{M} . \mathrm{Six}^{3}$ to the discount rate within the optimal pollution management is discussed. The references ${ }^{4-5}$ show: pollution control incentive and monitor problems are indeed important issues of environmental management. After analysis, we select are important indicators in pollution control: pollution control quality, pollution-control costs and on-time completion rate, setting a model of 4 sets of variable factors, and based on the theory of grey correlational analysis to study, concluded that indicators of relative importance. Expect to give reference for management incentive and monitor pollution-control behavior.

\section{Pollution Control Variables}

Incentives for pollution control and monitoring are based on indexes many more important indicators in practical application. They are pollution control quality, pollution-control costs and on-time completion rate. For simple and practical terms by these 3 common indicators primarily set model 4 sets of variable factors: the first group is the quality factor in time; the second group is the quality factor; a third group finished factor; the fourth group is the pollution control cost factor. Definition and analysis are as below. 
Quality on time factor. Refer to enterprises for pollution-control work by prior signed a contract with the management point and pollution control under the condition of quality of actual pollution control project items divided by the number specified in the contract in accordance with the appropriate conditions the percentage of rates. Permits to carry out pollution control can be scheduled, durability and security contracts, management issues of concern. In view of this, quality on time factor can represent polluters on the level of pollution control the overall performance of the contract.

Quality factor. Polluters according to the contract time quality and pollution control actually completed pollution control projects by dividing the number of time points should be stipulated in the contract rate of percentage of completed projects. This variable from the quality point of view permits of pollution control pollution control work, but also reflect the polluters on the level of performance of the contract.

Completion rate factor. Permits complete pollution control projects point in time by dividing the actual number of completed projects stipulated in the contract as stipulated in the contract should rate as a percentage of the number of completed projects. Pollution-control time of harsh conditions, completed number of items must be subject to rigorous challenges. It is to a certain extent on the level of project completion time description.

Pollution control cost factor. That permits complete costs of pollution control projects, which are the main polluters to meet cost commitments reflect. It is important to note, most of these 4 factors to consider these accepted and recognized the 3 more important indicator, in addition, in order to simplify the analysis assumes that firms only for the same type of pollution control projects (such as waste water control project).

The four groups in reality, the value of the variable can be calculated. Because on each a sewage enterprise completed of each a control dirt project, management authorities on control dirt project number, and control dirt quality, and cost and time is very care of, its also requirements sewage enterprise of control dirt sector on such of data has records, through these data on can seeking out on time quality completed rate, and quality completed rate, and on time completed rate and control dirt cost, specific numerical.

\section{Sewage Grey correlational Analysis Model of Enterprise Pollution Control Index}

Suppose that quality on time factor as $w_{0}(k)$, quality factor as $w_{1}(k)$, completion rate factor as $w_{2}(k)$, pollution control cost factor as $w_{3}(k)$ and set $k=1,2, \mathrm{~L} ; i=1,2,3$. Single proposition of Multipole analysis of grey system research for example. It means only a proposition, the polarity of individual sequences are not completely identical systems. System here is one of the few not sure system, which consists of a number of the original sequence. Sequence is a form of less data, information, and it is a way to describe system behavior. Suppose the original series set $\bar{\omega}$ consists of four sequences, namely, quality rates on time sequence ${ }^{w_{0}}$, quality rate of sequence ${ }^{w_{1}}$, completion rate for subsequences $w_{2}$, pollution control cost subsequence ${ }^{w_{3}}, \bullet$ is the change, then:

Definition 1 Suppose subset $X=\left\{X_{i} \mid i=1,2,3\right\}$ grey relating the set of factors (refers to factors with accessibility, comparability and polar). If $X=\bullet \bar{\omega}$, then $\bullet$ is grey correlation change, $\bullet=\{I N I T, A V G, I N V L\}$, INIT is initialized, AVG is equalization, INVL is interval. $X_{0}$ is the quality factor on time sequence, $X_{1}$ is the quality factor sequence, $X_{2}$ is the completion rate for factor 
sequence, ${ }^{X_{3}}$ is the emissions cost factor sequence. Here, the factor is the change of sequence. The indicator is the concrete expression of the proposition. And the proposition is content independent and complete tasks.

Definition 2 Suppose that $X_{0}$ is the specified reference sequence. And $\Delta_{01}=\left\{\Delta_{01}(1), \Delta_{01}(2), \mathrm{L}, \Delta_{01}(n)\right\}, \Delta_{01}(k)=\left|X_{(0)}(k)-X_{(1)}(k)\right|$. Then $\Delta_{01}$ is the information quality factor difference sequence. And it is the difference between quality factor and the sewage discharge factors measure. Let $\Delta_{02}=\left\{\Delta_{02}(1), \Delta_{02}(2), \mathrm{L}, \Delta_{02}(n)\right\}, \Delta_{02}(k)=\left|X_{(0)}(k)-X_{(2)}(k)\right|$. Then $\Delta_{02}$ is the completion rate for factor information sequence. And it is a time measure of the difference between the factor and the sewage discharge factors. Let $\Delta_{03}=\left\{\Delta_{03}(1), \Delta_{03}(2), \mathrm{L}, \Delta_{03}(n)\right\}$, $\Delta_{03}(k)=\left|X_{(0)}(k)-X_{(3)}(k)\right|$. Then $\Delta_{03}$ cost factors for message sequence. And it is the measurement of difference between cost factor and sewage discharge factors.

Definition 3 Suppose $\Delta(\max )=\max _{i} \max _{k} \Delta_{0 i}(k), \Delta(\min )=\min _{i} \min _{k} \Delta_{0 i}(k)$. Then $\Delta(\max )$ is the on time quality rate of environmental parameters. $\Delta(\min )$ is the down environment parameters for quality and on time rate. Here, the environment is a comparative neighborhood region. The upper and down information parameters represent the maximum and minimum values on difference of upper and down environmental parameters representative factors.

Definition 4 Suppose that $\zeta$ is a real number between 0 and 1 . Then $B_{D I}=\left\{\Delta_{0 i}, \Delta(\max ), \Delta(\min ), \zeta\right\}$ is the differential information box. It can be used to reflect and calculate the differential information measurement between factors in grey correlation system.

Definition 5 Suppose $r\left(X_{(0)}(k), X_{(i)}(k)\right)=\left[\max _{i} \max _{k} \Delta_{0 i}(k)+\zeta \min _{i} \min _{k} \Delta_{0 i}(k)\right] \div\left[\Delta_{0 i}(k)+\zeta \max _{i} \max _{k} \Delta_{0 i}(k)\right]$. Then $r\left(X_{(0)}(k), X_{(i)}(k)\right)$ quality on time completion rate grey correlation coefficient, which means the association degree between the impact factors and the baseline factors in system, and which is the reflection of relevant influence. It embodies the overall comparison of the reference system and the test. And it reflects the combination and sublimation of point set topology and metric spaces.

Definition 6 Suppose $\xi_{01}=\left\{r\left(X_{(0)}(1), X_{(1)}(1)\right), r\left(X_{(0)}(2), X_{(1)}(2)\right), \mathrm{L}, r\left(X_{(0)}(n), X_{(1)}(n)\right)\right\}$, $\xi_{02}=\left\{r\left(X_{(0)}(1), X_{(2)}(1)\right), r\left(X_{(0)}(2), X_{(2)}(2)\right), \mathrm{L}, r\left(X_{(0)}(n), X_{(2)}(n)\right)\right\} \quad, \quad \xi_{03}=\left\{r\left(X_{(0)}(1), X_{(3)}(1)\right)\right.$, $\left.r\left(X_{(0)}(2), X_{(3)}(2)\right), \mathrm{L}, r\left(X_{(0)}(n), X_{(3)}(n)\right)\right\}$. Then $\xi_{01}$ is the grey correlation set of completion rate for quality and on-time quality finish rate. It reflects the correlation between the quality of pollution-control factor and emission performance factor. $\xi_{02}$ is the grey correlation set of on time completion rate and quality finish rate. It reflects the correlation between the time factor and emission performance factor. $\xi_{03}$ is the grey correlation set of pollution control cost and quality finish on time rate. It reflects the correlation between the cost factor and emission performance factor.

$\begin{array}{lll}\text { Definition } & 7 \quad \text { Suppose } \quad r\left(X_{(0)}, X_{(1)}\right)=\frac{1}{n}\left[r\left(X_{(0)}(1), X_{(1)}(1)\right)+r\left(X_{(0)}(2), X_{(1)}(2)\right)+\mathrm{L}+r\left(X_{(0)}(n), X_{(1)}(n)\right)\right] \\ \left.r\left(X_{(0)}, X_{(2)}\right)=\frac{1}{n} r\left(X_{(0)}(1), X_{(2)}(1)\right)+r\left(X_{(0)}(2), X_{(2)}(2)\right)+\mathrm{L}+r\left(X_{(0)}(n), X_{(2)}(n)\right)\right] & r\left(X_{(0)}, X_{(3)}\right)=\frac{1}{n}\left[r\left(X_{(0)}(1), X_{(3)}(1)\right)+r\left(X_{(0)}(2), X_{(3)}(2)\right)+\mathrm{L}+r\left(X_{(0)}(n), X_{(3)}(n)\right)\right] . \text { Then }\end{array}$. 
$r\left(X_{(0)}, X_{(1)}\right)$ is the grey correlation degree between quality completion rate and the quality on time rate. It embodies the measurement of the impact of pollution-control quality factor on polluters' performance. $r\left(X_{(0)}, X_{(2)}\right)$ is the grey correlation degree between complete rate and quality on time rate. It reflects the measurement of the impact of the time factor on polluters' performance. $r\left(X_{(0)}, X_{(3)}\right)$ is the grey correlation degree between pollution control cost and quality on time rate. It reflects the measurement of the impact of the cost factor on polluters' performance.

Definition 8 Suppose $\psi\left(X_{(0)}, X_{(1)}\right)=r\left(X_{(0)}, X_{(1)}\right) \div\left[\sum_{i=1}^{3} r\left(X_{(0)}, X_{(i)}\right)\right], \psi\left(X_{(0)}, X_{(2)}\right)=r\left(X_{(0)}, X_{(2)}\right) \div\left[\sum_{i=1}^{3} r\left(X_{(0)}, X_{(i)}\right)\right]$. $\psi\left(X_{(0)}, X_{(3)}\right)=r\left(X_{(0)}, X_{(3)}\right) \div\left[\sum_{i=1}^{3} r\left(X_{(0)}, X_{(i)}\right)\right]$. Then $\psi\left(X_{(0)}, X_{(1)}\right)$ is the relative weight measurement of the quality on time rate for evaluating of sewage enterprise's quality on time performance. It reflects the relative importance of the pollution control quality attribute influencing sewage enterprise's performance. $\psi\left(X_{(0)}, X_{(2)}\right)$ is the relative weight measurement of the on time rate for evaluating of sewage enterprise's quality on time performance. It reflects the relative importance of the time attribute influencing sewage enterprise's performance. $\psi\left(X_{(0)}, X_{(3)}\right)$ is the relative weight measurement of the pollution control cost for evaluating of sewage enterprise's quality on time performance. It reflects the relative importance of the cost attribute influencing sewage enterprise's performance.

\section{Data Samples}

Numerical calculation and analysis of the cases are given here. Set rate unit is percentage, numerical unit is ten thousand yuan, $\zeta$ 's value is 0.5 . Given the raw data: $w_{0}=(0.803,0.852,0.869,0.901)$; $w_{1}=(0.822,0.875,0.888,0.959) \quad ; \quad w_{2}=(0.873,0.786,0.870,0.789) \quad ; \quad w_{3}=(1.21,1.25,1.18,1.28)$ According to the model defined in this paper, the relative measurement of sewage enterprise attribute can be determined: $\psi\left(X_{(0)}, X_{(1)}\right)=0.425634 ; \psi\left(X_{(0)}, X_{(2)}\right)=0.267236 ; \psi\left(X_{(0)}, X_{(3)}\right)=0.307130$. Thus, the influence of quality completion rate on polluters to fulfill on time quality rate is the most, so the weights should be most. The influence of pollution control cost to quality on time rate of polluters' performance is smaller, so the weight should be smaller. The completion rate hast he minimal effect on polluters to fulfil quality on time completion rate, so the weight should be minimal.

\section{Acknowledgement}

This work was financially supported by the Science and Technology project of Liaoning Science and Technology Bureau of 2017; project (20170540439); the project's name: The pollution controlling model system's construction based on middle-micro scale.

\section{References}

1.JoschkaGerigk, IanA.MacKenzie, MarkusOhndorf. A Model of Benchmarking Regulation: Revisiting the Efficiency of Environmental Standards. Environ Resource Econ (2015) 62:59-82 
2. Kimitoshi Sato. Anthropogenic Climate Change in an Integrated Energy Balance Model of Global and Urban Warming. Journal of Economic Structures (2014) 3-9

3. Milan časny, Emanuele Massetti, Jan Melichar, Samuel Carrara. Quantifying the Ancillary Benefits of the Representative Concentration Pathways on Air Quality in Europe. Environ Resource Econ (2015) 62:383-415

4. Georg Hirte, Stefan Tscharaktschiew. Optimal Fuel Taxes and Heterogeneity of Cities. Rev Reg Res (2015) 35:173-209

5. Marcelle C. McManus, Caroline M. Taylor, Alison Mohr, Carly Whittaker, Corinne D. Scown, Aiduan Li Borrion, Neryssa J. Glithero, Yao Yin. Challenge clusters facing LCA in environmental decision-making--what we can learn from biofuels. Int J Life Cycle Assess (2015) 20:1399-1414 